\title{
CarryMe: Drone Delivery System for Flooded Area
}

\author{
T. L. H. Gammedda \\ Sri Lanka Institute of \\ Information Technology
}

\author{
W. V. Dilushika \\ Sri Lanka Institute of \\ Information Technology
}

\author{
D. C. Gunasekare \\ Sri Lanka Institute of \\ Information Technology
}

\author{
W. C. I. D. Silva \\ Sri Lanka Institute of Information Technology
}

\author{
S. G. S. Fernando \\ Sri Lanka Institute of Information Technology
}

\begin{abstract}
We live in a world where natural disasters keep increasing frequently and severely. Sri Lanka being an island, flood is considered as the most frequently affected natural hazard in Sri Lanka. When such situations arise, normally boats and helicopters are used to provide necessary goods to affected people. Less efficiency, inaccessibility to some areas, need of more human effort and uncertainty of the delivery are the major issues of these two methods. "CarryMe" is the drone delivery system which can be used to provide better disaster recovery by resolving these issues. Collision avoidance algorithm, obstacle detection algorithm, video streaming and autopilot are the main functionalities of this implemented system. A flooded area can be identified using a map through the web application of CarryMe. Travelling Salesman Problem algorithm was used to find the optimal path. The web application facilitates the user to handle several drones at the same time as well which is a special feature of this drone system. Special programming techniques like Arduino, Node.js and micro service architecture will be used in programming and sensors like ultrasonic is used to detect the distance to the obstacle. Deliveries that can be done efficiently in any inaccessible area without any trouble with low cost and low human effort can be listed as the benefits of CarryMe as well as the drone controlling interface which user- friendly and it can serve several people using several drones at the same time.
\end{abstract}

\section{General Terms}

Algorithm, disaster recovery, programming, web application

\section{Keywords}

Arduino, GPS, ultrasonic sensors, GSM module, Node.js, Video streaming

\section{INTRODUCTION}

Tsunami, floods, landscapes are the well-known disasters which are normally occurred in Sri Lanka. As Sri Lanka is a developing country, this will result in the heavy losses to the country and the people. Sri Lanka is associated with major floods in the two monsoons. On 15th may 2016, 22 districts were widespread with flooding because of a tropical storm. At least 104 people are known to have died because of this disaster [1].The most adjacent disaster has occurred on 30th may in this year. It also resulted by affecting 545283 people, 768 fully damaged houses and 5869 partially damaged houses [2].

Problem is, even though the disasters happen in Sri Lanka for a long time, still, there is no proper coordination to relief the people. Vehicles, boats and helicopters are current distribution methods of goods. These two methods is not efficient because it needs more cost, more human effort and sometimes boats could not be able to reach some areas. As a solution to this problem drone was implemented. It is capable of delivering goods at low cost for each affected area. Drone technology is rapidly evolved in modern world. It has begun used in Security, surveillance, Aerial Photography and Inspection.

The major objectives of the project is given below,

1. To develop a drone to deliver goods for flooded areas.

2. To develop a collision detection algorithm to avoid collision.

3. To develop an obstacle detection algorithm to detect obstacles.

4. To develop a web application and indicate the flooded areas using a map.

Furthermore this research paper discussed about the Background, Methodology used to implement the system and finally about the discussion and conclusion of the overall system.

\section{BACKGROUND}

There are many applications have been developed using drone technology. Agricultural surveillance, disaster monitoring, scientific research and exportation and aerial photography are some of the applications where the drone technology has been equally involved. Among those, drone delivery is the one of the interesting area focused by the researchers.

\subsection{Obstacle detection and collision avoidance}

Onboard sensing and onboard processing power are the factors of limiting the autonomy of the obstacle detection. Hardware setup and processing pipeline is allowed to fully autonomous UAV to detect obstacles in almost all directions .3D laser scanner which is capable of providing an omnidirectional view with the spars measurements at a lower frame rate. To overcome this issue, a pair of stereo cameras have connects that is capable of providing additional sparse measurements at high frame rate. To detect the obstacles which have difficulties with detecting and the small in sizes such as tree branches or cables at high frame rates as well as transparent obstacles ultrasonic sensors are used by them. The cost of this product is very high [4].

It is no longer necessary for firefighters or other support personnel to enter the danger zone user-friendly analyze the situation. The drones can now fulfill this role. The modular 
payload system allows various devices to be attached to the drone. The smoke composition of a fire can be communicated using a gas measurement device. It is a greater chance of firefighting teams to aware about the situation. Using obstacle detection and collision avoidance technology drone enter the danger zone to consume and thermal image camera can assist in searches for missing persons who are lay down in forests, fields and etc. [7].

Obstacle detection and collision avoidance technology becomes an emergency tool for firefighters and disaster controllers over observation and exploration. As well as a small flying machine can help humans in their daily work. In the obstacle detection module, redundant ultrasonic sensors are used to increase detection resolution and sensor data reliability. The collision avoidance module divides the area around the quadcopter dependent on the measured distance into three zones for each [6].

\subsection{Video streaming}

"Public Spaces Mobile Video Chat" provides a parallel experience using the technology of video streaming. Video streaming, being the best in navigation creates a very intimate experience with the remote partner. But this can lead to distractions from the real world. For this Probe technology, Video Streaming is used along with the "Shared Geocaching". Issues were raised around privacy, safety, navigation and 'micro' shared experiences [5].

\subsection{Drone autopilot system}

As an answer to the traditional time consuming and expensive drone delivery system, viable drone based autonomous delivery system was designed and implemented. Full autonomous carry and delivery static route navigation, and single touch point interaction was the main requirement of this system. Autopilot drone \& (E-O) Electro-optical identification technology is advanced enough that it can potentially hostile target at an attitude 5000 feet and also provide real-time imagery to a ground control operator [8].

Aerial photography is another field which many types of research have been focused on. The autopilot quadcopter is capable of taking the aerial photography in agricultural applications. EK-LM4F120XL module acts the role of the main control unit in order to control the autopilot system.NAZA-M light flight control system with the GPS module was used to build the overall system. In order to take clear aerial photos 5Mpixel camera was used and the images which were taken from the camera processed with the NDVI technique which is capable of providing the details on the photosynthesis status of rice leaves. This system can be used to evaluate the stress of the plant and the health. Stability and flight time are the issues related to the system which was introduced by them [3].

\subsection{Arduino technology related to drone}

Drone is formally known as Unmanned Arial vehicle (UAV). Essentially drone is an aircraft without a human pilot onboard a flying robot. Today most drone systems use Arduino technology because it is easy to use. The most popular and common Arduino board is Arduino Uno. It is a microcontroller board based on the Atmega328. It has 14 input/output pins, 6 analog inputs, $16 \mathrm{MHz}$ ceramic resonator, USB connection, power jack and a reset button. It is needs connected to the computer with USB cable or powers it with AC-to-DC adapter to get started. The power source is selected automatically. The adapter can be connected by plugging a $2.1 \mathrm{~mm}$ center-positive plug into the board's power jack. If using more than $12 \mathrm{~V}$ the voltage regulator may overheat and damage the board. The recommended range is 7-11V [9].

\section{METHODOLOGY}

Prototype methodology is selected to implement the system and all the activities done for each and every stage is discussed below.

\subsection{Planning}

A feasibility study was conducted mainly under three categories. They are time, cost and scope. Even though this is a research project, it needed to complete within a very limited time period. Is it possible? Then check whether the project was able to complete with the limited budget which the project team had. The technologies, hardware and software components which are needed to implement the "CarryMe" system were new to the research team. Therefore check whether project team has interest and ability to adapt or learn new frameworks or other new technologies. After conducting these feasibility study project team identified this project was enough feasible to start implementing in the planning phase.

\subsection{Requirement gathering \& analysis}

The requirement gathering was done using primary data and secondary data. As primary data interviews were conducted with the Disaster Recovery Center. The results of interviews were, current methods like boats, helicopters and trucks are not possible to reach every area because some areas are inaccessible due to the severe damage is done by the flood. Therefore proper method like drone which is very small compared to other delivery methods is the most suitable way to deliver goods in order to rescue humans life was the final conclusion of the interviews. As the secondary data project team went through several research papers. In this, research team researched for existing similar systems and analyzed their functionalities, to better understand the methodologies used. Information regarding Obstacle detection, collision avoidance and autopilot techniques of the drone were analyzed using existing research as well.

\subsection{Designing}

The drone structure, collision detection algorithm, obstacle avoidance algorithm, autopilot system and interfaces are designed as main component of the "CarryMe" system in this phase. Hardware parts needed to implement the drone structure was contained several varieties and it is very difficult to find the best ones with the limited budget. Therefore first small designing plan was made for the behavior of the ultrasonic sensors, bmp sensors, flight controller, PWM generator, A6 GSM module and how they were placed in the drone frame in more organizing way.

Figure 1 is illustrated the Architecture diagram. It is explained about main relationships between the user and other system components. Operator can access to the web application through the web browser which is at the personal computer. This web application is hosted in separate server. Location details provided to the web application reach the drone control unit through the server. Details related to different drones like videos, good delivery details are gone to the database through the server and whenever operator request these details those are traveled from database to server and finally server to web application which is at the personal computer. Now every detail can be viewed by the user. Like this each and every component is worked together to deliver fully functional system. 


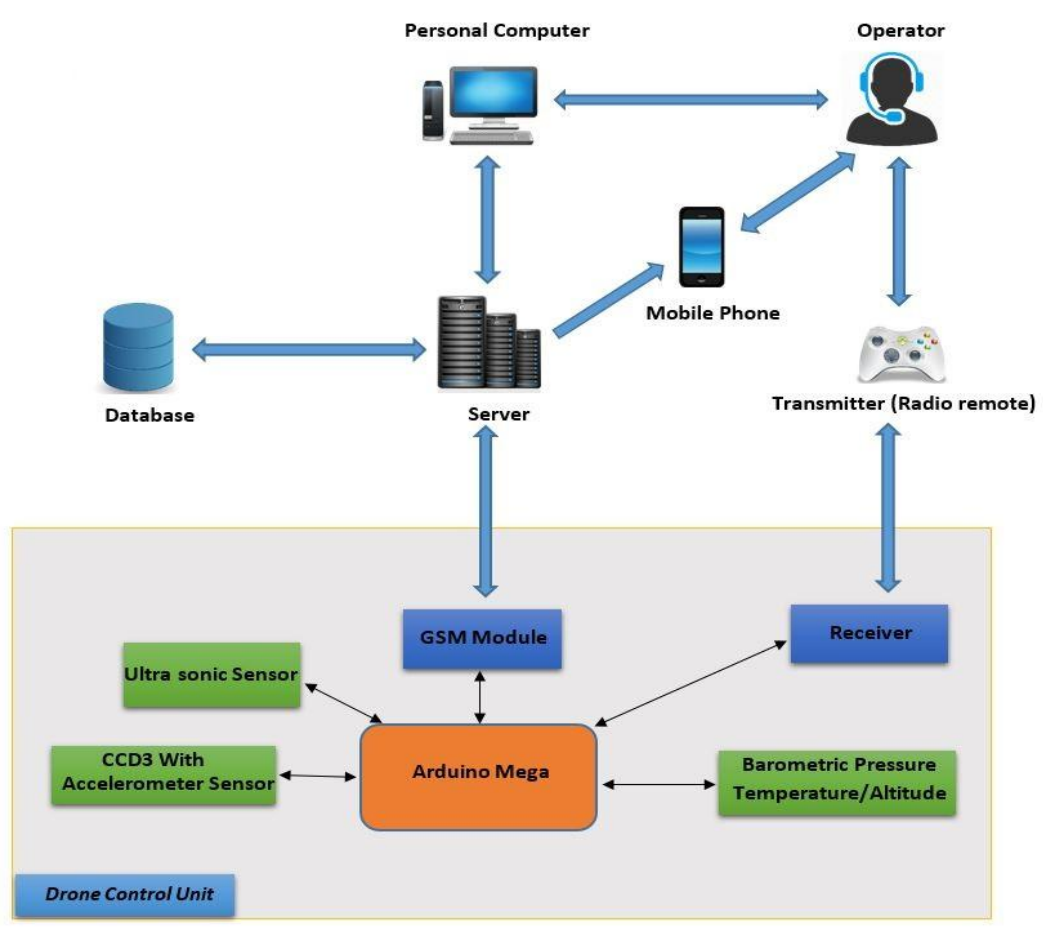

Figure 1: Architecture Diagram

\subsection{Implementation}

As a first step for this phase drone was implemented by combining all the hardware parts as shown in figure 2 . The System is developed as separate units for the purpose of developing the code. The requirements are met by developing the system separately as smaller units and integrating to form one full system. Arduino language was used to implement obstacle detection algorithm, collision avoidance algorithm and autopilot using the Arduino IDE. The website was developed using HTML and CSS. Multiple drones controlling and all the other main functions are implemented using node.js framework (JavaScript framework).To handle the video streaming mobile application was developed using react native language.

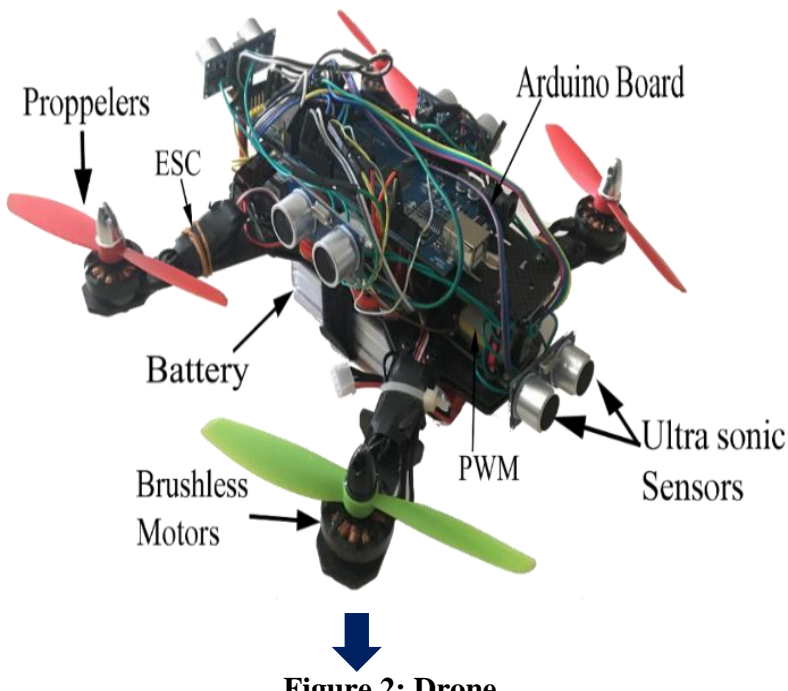

Figure 2: Drone

\subsection{Testing}

All testing was done in the "prototype level". The two main testing methodologies used were Black-Box testing and WhiteBox testing. Black box testing was done to ensure all the functionality of the system is working as specified. Structure of the functionalities is checked using white box testing.

\section{RESULTS \& DISCUSSION}

The "CarryMe" System has three major components. They are drone, web application and mobile application. Web application is used to control the drone and mobile application is used for video streaming. To enable the communication between the three major components GSM module is used.

To control multiple drones, a drone name is used. If there are multiple drones all the drones can be searches according to their names. Once user select some specific drone to the delivery and click the search button always redirect to its corresponding controller interface. Controller interface is the one which facilitate user to select the flooded area needed to be traveled. Always drone name is added at the end of the URL in controller interface. This is help to user to identify the drone which is used for delivery and make sure it is the one which selected by him at the previous step. All the location details, videos are recorded according to the id (primary key of the drone table) in the database. This process is illustrated by figure 3 and figure 4 .

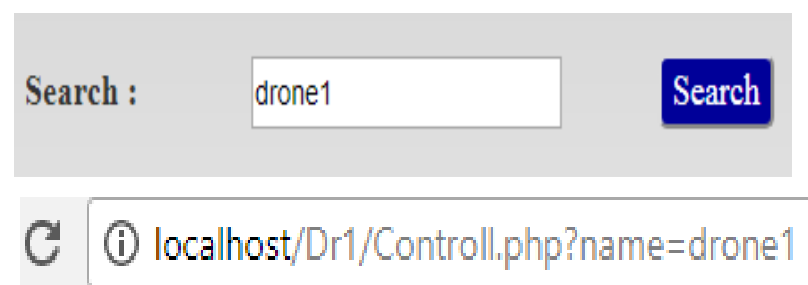

Figure 3: Search by Name_1 


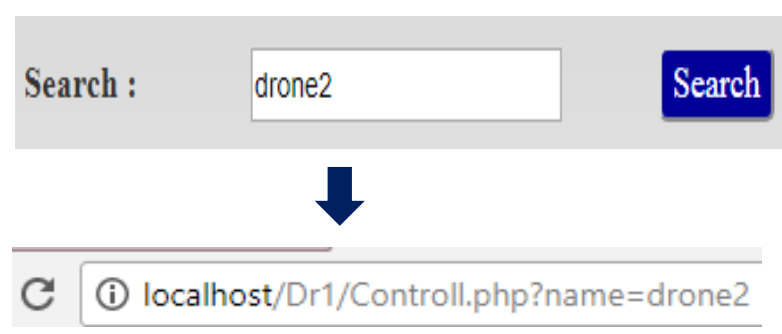

Figure 4: Search by Name_2

To detect obstacles in more accurate way six ultrasonic sensors were used. These sensors are placed on top, bottom, front, rear, right and left side of the drone. First thing is to find the distance to the obstacles. But the problem is ultrasonic sensor always give the readings in milliseconds. Therefore small calculation was used to convert this into centimeters as shown in figure 6 Then consider all the possibilities obstacle can be occurred in six sensors within the range of $200 \mathrm{~cm}$ from the drone's current location and guide the drone into correct direction to avoid the collision as shown in figure 5 .

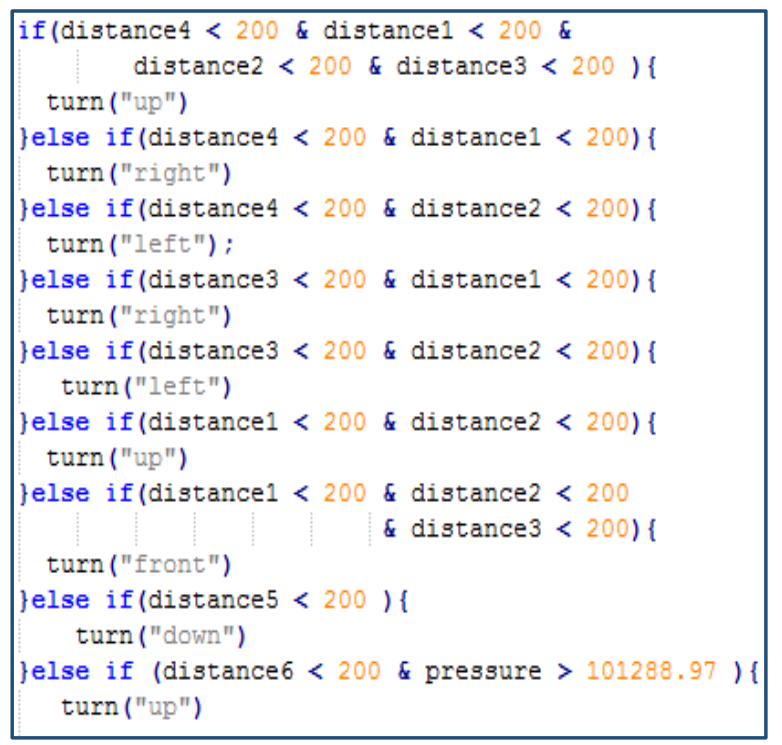

Figure 5: Obstacle Detection

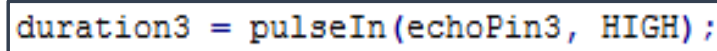

Figure 6: Distance Calculation

Collision avoidance was done using the parameter passed by the obstacle detection algorithm. This parameter contains the correct turning direction to avoid the collision. Drone is controlled by the four channels. Cannel one is for up and down, channel two is for left, channel three is for right and channel four is for left right rotation. As shown in figure 7 pwm.setPWM pass the relevant PWM values to PWM generator to avoid collision.

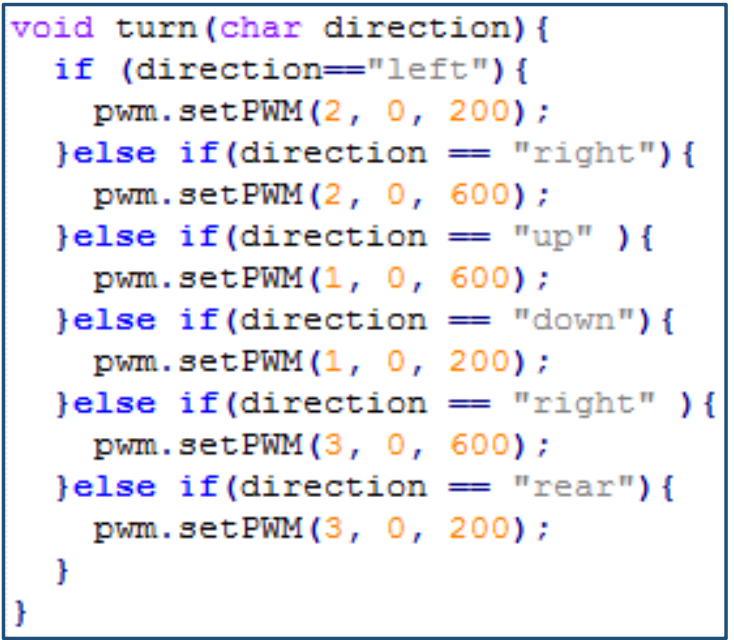

Figure 7: Collision Avoidance

To indicate the flooded area map which is in the web application is used. If drone needs to travel several areas, travelling salesman algorithm (find the shortest path for each destination) is used to identify the optimum path. Then Latitude and longitude values related to these paths are pushed into a stack according to the resulted order. Current location details are always stored at the zero index of the stack because drone should be returned to the source after done the delivery successfully.

To find the optimal path need to pass the distance matrix of all the selected places as an input to travelling salesmen problem algorithm .Figure 8 is illustrated the TSP function. To do that mainly there are three arrays was used. Latitude and longitude values of all the places are in the arr_lat[] and arr_long[] arrays. Dist_array[] is a large array which contain the whole distance matrix. First for loop is used to design the rows and second for loop is designed the columns. In other words it helps to calculate the distance from one place to every other place. If user selects 5 places, need to consider about 25 calculations to find the least cost path to every other nodes or places.

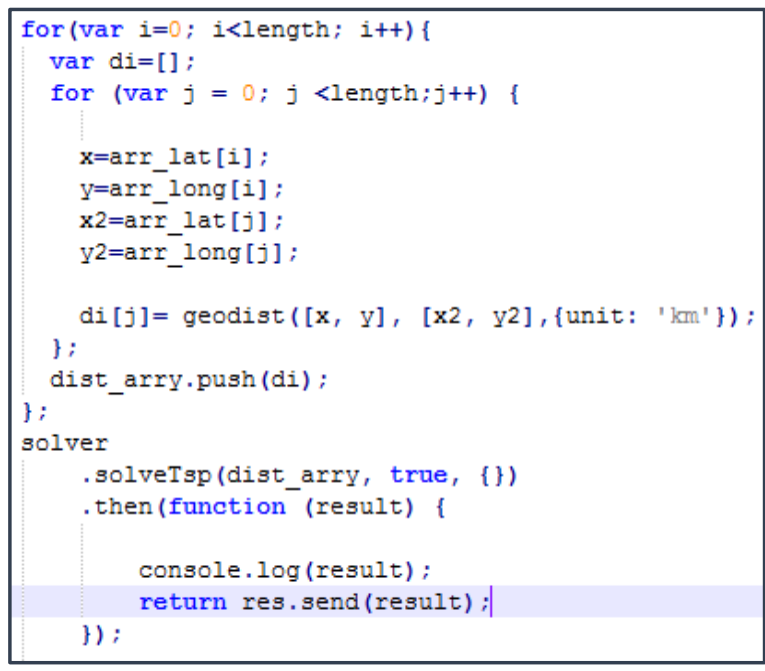

Figure 8: TSP function (Travelling Salesman Problem)

Current, previous and target location details are mainly used to make the drone autopilot. Readings related to the current latitude and longitude is given by the GPS module. Target latitude and longitude details were given by the web application pass through GSM module. Initially current and previous 
locations are at the same point. As shown in figure 10, need to calculate the tan values between current and previous and between current and the target. Compare these two values as shown in figure 9 using if else condition and guide the drone to fly into the correct direction. If Value of M1 is greater than M2 drone should turn to the right. If it is other way round should turn into left. But M1 and M2 values are equal no need to change the path of the drone. According to these directions drone is able to reach its destination autonomously.

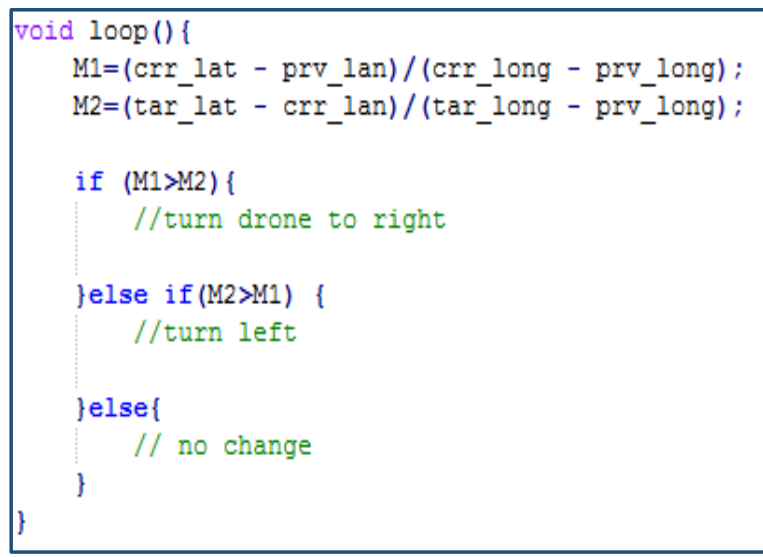

Figure 9: Autopilot function

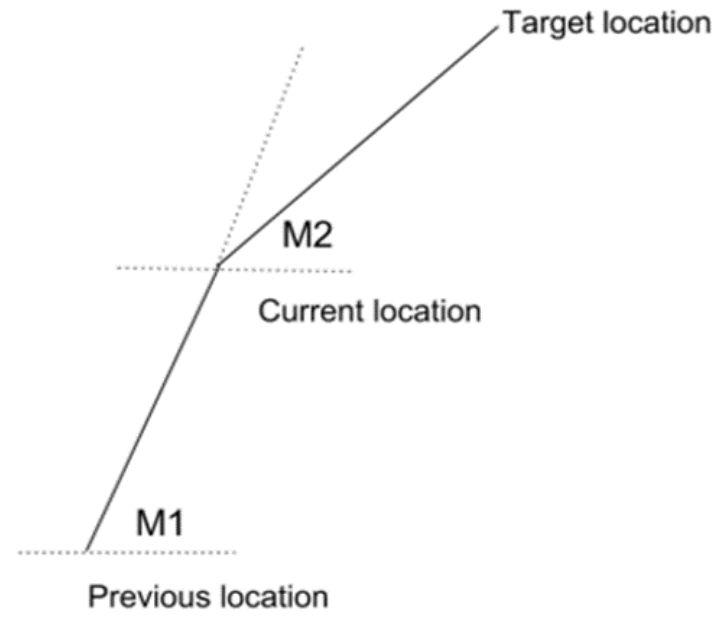

Figure 10: Autopilot Calculation Design

Video Streaming was done through the mobile application. This mobile application is compatible with both Android and IOS versions. Name of the DRONE helps to stream videos over multiple drones. QR codes are used to identify the camera and drone when they are kept in separately. Therefore drone can do video streaming using any other camera. Mobile app also able to read the QR codes in the drone and camera.

\section{CONCLUSION}

"CarryMe" system was developed to give an effective recovery process to the affected people by the flood. This is an autonomous drone delivery system which can easily reach to any area without any trouble. When a disaster is happen lot of goods need to be transported, so specialty of this system is, at a time several drones can be handling using one website. "CarryMe" is capable of delivering several areas using one visit as well. This innovated system is facilitating to get a close and clear view of the affected area through the mobile application.
System uses six ultrasonic sensors which are placed front, rear, left, right, up and down sides of the drone to detect obstacles in more accurate way. Once these sensors detect the obstacles, PWM generator is used to send the signals of the direction to drone without any delay to avoid the collision. Drone is travelled to particular destination according to the latitude and longitude values provided by the website. If drone need to travel to three locations location details are stored in a stack. Once it reaches to one destination it checks the stack to check whether there are any other places to visit. If there are no other places it return to the current location which is stored in the zero index of the stack. To land the drone without causing any harm, two SMS are sent to the operator's phone. First SMS is sent $1 \mathrm{~km}$ away from the destination and second SMS is sent within $1 \mathrm{~km}$ to the destination. All these testing were done successfully. Therefore system is accurate and reliable to use.

With all this functionalities, this system has some limitations as well. This system is completely dealing with the internet, so to operate properly system needs good internet signal strength. If it is not, system can stop the operation. This system is not capable of travelling to long distance because of the less battery power, less motor speeds and other hardware component capacity issues. When situation like bad whether condition is occurred this system cannot handle itself because of the less battery capacity, insufficient esc power and the used sensors not capable enough to give 100 percent accurate readings.

"CarryMe" is currently compatible with only web sites. In future drone controlling system can be developed as a mobile application as well. This is only designed for short distances delivery but the inbuilt architecture is sufficient enough for long distance as well. So using efficient hardware can make this system to travel long distance. When landing drones to distribute the goods, this system still used inbuilt programming. So this system can develop to distribute goods using Artificial Intelligence in future.

\section{ACKNOWLEDGEMENT}

Research group would like to thank Sri Lanka Institute of Information technology for given to explore areas of education that would never have been possible. Secondly, the supervisor S.G.S.Fernando for guiding the group in continuous way to bring this a success and all the other people who help to this project deserves grateful thanks.

\section{REFERENCES}

[1] "Disasters",ReliefWeb,2017.[Online].Available:https: //reliefweb.int/disaster/fl-2016-000050-lka.[Accessed: 24- Sep- 2017].

[2] 2017.[Online].Available:https://www.iom.int/sites/def ault/files/country/docs/srilanka/IOM-SL Displacement-Map.pdf.[Accessed: 24- Sep- 2017].

[3] 2017.[Online].Available:http://www.arpnjournals.org/ jeas/research_papers/rp_2016/jeas_0416_3997.pdf.[A ccessed: 19- Mar- 2017].

[4] 2017.[Online].Available:http://www.cv.foundation.or g/openaccess/content_cvpr_2015/papers/Rozantsev_F lying_Objects_Detection_2015_CVPR_paper.pdf.[Ac cessed: 19- Mar- 2017].

[5] 2017.[Online].Available:https://static.googleuserconte nt.commedia/research.google.com/en//pubs/archive/4 3118.pdf.[Accessed: 19- Mar- 2017]. 
[6] 2017.[Online].Available:http://www.mdpi.com/20751 702/2/1/13/machines-02-00013-pdf-vor.pdf [Accessed: 19- Mar- 2017].

[7] 2017.[Online].Available:http://www.montenegros.de/ sergio/public/microdromes12collisionavoidance.pdf.[ Accessed: 19- Mar- 2017].
[8] AerWaze Technologies Help Can Avert Drone AviationDisasters",Pressreleasejet.com,2017.[Online] .Available:https://pressreleasejet.com/news/aer-wazetechnologies-help-can-avert-drone-aviationdisasters.html.[Accessed: 19- Mar- 2017].

[9] 2017.[Online].Available:http://ijaeit.com/Volume-2Issue-5-2016/3.pdf.[Accessed: 20- Mar- 2017]. 